\title{
TABLE DES SIGLES
}

ABN Autorité du bassin du Niger

ADN Acide désoxyribonucléique

AEP Alimentation en eau potable

AFD Agence française pour le développement

Afssa Agence française de la sécurité sanitaire des aliments

Afsse Agence française de sécurité sanitaire environnementale

Agrhimet Centre du CILSS à Niamey, Niger, pour la formation dans le domaine de l'agro-écologie (agroclimatologie, hydrologie, protection des végétaux)

AMCOW African Ministerial Conference on Water

ARN Acide ribonucléique

AQEM Aquatic Quality Evaluation Method

$\mathrm{AVCl} \quad$ Années de vie corrigées du facteur invalidité, DALY en anglais

AWTF African Water Task Force

CEH Centre for ecology and hydrology, Wallingford, Royaume-Uni

Cemagref Centre national du machinisme agricole, du génie rural, des eaux et des forêts

CILSS Comité inter-États de lutte contre la sécheresse au Sahel

Cipel Commission internationale de protection des eaux du Léman

Cirad Centre de coopération internationale en recherche agronomique pour le développement

CME Conseil mondial de l'eau

CNR Compagnie nationale du Rhône

CNRM Centre national de recherches météorologiques, Toulouse

CSHPF Conseil supérieur d'hygiène publique de France

DALY Disability Adjusted Life Years

DCE Directive cadre européenne sur l'eau 
Ddass Direction départementale de l'action sanitaire et sociale

DDT Dichlorodiphényltrichloroéthane, un insecticide

DG Direction générale (à la Communauté européenne à Bruxelles)

DGS Direction générale de la Santé, ministère de la Santé, Paris

DHI Danish Hydological Institute

DJT Dose journalière tolérable

Drass Direction régionale de l'action sanitaire et sociale

Drire Direction régionale de l'industrie, de la recherche et de l'environnement

DVGW Deutscher Verein des Gas und Wasserfaches

EDF Électricité de France

EIER École inter-États d'ingénieurs de l'équipement rural à Ouagadougou, Burkina-Faso

ENSO El Niño Southern Oscillation

EPA voir USEPA

ESCAP Comité économique et social pour l'Asie-Pacifique (http://www.carnetsduvietnam.com/web/infos/mars2001.htm)

ETSHER École inter-États des techniciens supérieurs de l'hydraulique et de l'équipement rural, Kamboinsé, Ouagadougou, Burkina-Faso

FAO Food and Agriculural Organisation (Nations unies)

FMI Fond monétaire international

GDF Gaz de France

GEA Gastro-entérites aiguës

GES Gaz à effet de serre

GESI Initiative mondiale d'assainissement environnemental

Gicc Groupe interministériel sur les changements climatiques, Paris

Giec Groupe intergouvernemental sur l'évolution du climat

GIWA Global International Waters Assessment du PNUE

GMR Great Man-Made River (Grande rivière artificielle), Libye 
GRDC Global Runoff Data Center

GWSP Global Water System Project

G8 Groupe des huit pays les plus industrialisés

HACCP Hazard Analysis, Critical Control Points

ICARDA International Centre for Agricultural Research in the Dry Areas, Aleph, Syrie

Ifen

Institut français de l'environnement

IHE

Institute of Hydraulic Engineering, Unesco, Delft

IHS

Institute of Hydrological Sciences, Wallingford, UK, devenu maintenant $\mathrm{CEH}$

InVS Institut de veille sanitaire, ministère de la Santé, Paris

IPCC Intergovernmental Panel on Climate Change, Giec en Français

IRD Institut de recherche pour le développement, Paris

ITCZ Zone de convergence intertropicale

IWMI International Water Management Institute, Colombo, Sri Lanka

KIWA Keuringsinstituut voor Waterleidingartikelen, Organisme néerlandais de certification dans le domaine de l'eau

Lp Legionella Pneumophila

MEDD Ministère de l'Écologie et du Développement durable, Paris

MODB Matière organique bio-éliminable

MON Matières organiques naturelles

MNHN Muséum national d'histoire naturelle, Paris

Nepad New partnership for african development

NOAA National Oceanic and Atmospheric Administration, États-Unis

OGM Organismes génétiquement modifiés

OHRAOC Observatoire hydrologique régional de l'Afrique de l'Ouest et Centrale

OMD Objectifs du Millénaire pour le développement de l'ONU

OMM Organisation météorologique mondiale 
OMS Organisation mondiale de la santé

OMVS Office de mise en valeur du fleuve Sénégal

OMVG Office de mise en valeur du fleuve Gambie

ONG Organisation non gouvernementale

ONU Organisation des Nations unies

ORE Observatoire de recherche sur l'environnement

OSS Office du Sahara et du Sahel, Tunis

PAGER Programme d'alimentation en eau potable des populations rurales au Maroc

PED Pays en développement

PIB Produit intérieur brut

Piren Programme interdisciplinaire de recherche sur l'environnement du CNRS

PME Partenariat mondial pour l'eau

PMPOA Programme de maîtrise des pollutions d'origine agricole

PNB Produit national brut

PNSE Plan national santé environnement

PNUE Programme des Nations unies pour l'environnement

PPRI Plans de prévention des risques d'inondation

RATP Régie autonome des transports parisiens

RFF Réseau ferré de France

RMC River Mékong Commission

RTE Réseau de transport d'électricité

Sage Schéma d'aménagement et de gestion des eaux

SASS Système aquifère du Sahara septentrional

Schapi Service central d'hydrométéorologie et d'appui à la prévision des inondations

Sdage Schéma directeur d'aménagement et de gestion des eaux

SDT Syndrome de dysgénésie testiculaire 
SISE-Eau Système d'information santé environnement-eau

SNCF Société national des chemins de fer français

THF Tétrahydrofurane

UICF Union internationale des chemins de fer

UICN Union mondiale pour la nature, Gland, Suisse

USEPA United States Environmental Protection Agency

Unesco Programme des Nations unies pour la culture

Unicef United Nations International Children's Emergency Fund

$\mathrm{VIH} \quad$ Virus de l'immunodéficience humaine

Wateco Groupe de travail européen sur l'eau, dans le cadre de la DCE

Whycos Système mondial d'observation du cycle hydrologique (OMS)

WHO World Health Organisation, ov encore OMS en Français

WRC Water Research Centre, Royaume-Uni

WWAP World Water Assessment Programme

WWF World Water Forum ou aussi World Wildlife Fund 
